\title{
Noticias del nutritionDay NutritionDay 2020 en el contexto de la COVID-19
}

\author{
NutritionDay news \\ NutritionDay in the COVID-19 context \\ Notícias da nutritionDay \\ NutritionDay 2020 no contexto do IDVC-19
}

Angélica María Pérez, ND, MSc (c) ${ }^{1 *}$

https://doi.org/10.35454/rncm.v4n1.250

\begin{abstract}
El "Día de la Nutrición" (nutritionDay) es una iniciativa anual internacional en la que participan las instituciones de atención en salud con el fin de vigilar, evaluar y mejorar la situación nutricional de los pacientes. A través de la recolección de datos en un día, el nutritionDay permite obtener un mapa preciso de los indicadores del cuidado nutricional y de la prevalencia de la malnutrición en las instituciones hospitalarias, así como medir la disminución de la ingesta de nutrientes de acuerdo con los factores de riesgo por especialidad médica y con la estructura organizativa ${ }^{(1)}$. Esto se realiza mediante cuatro cuestionarios, los cuales están disponibles en más de 24 idiomas para que los pacientes y el personal de cada hospital puedan llenarlos con facilidad. Los datos recogidos son analizados de forma anónima y los resultados de cada hospital quedan a disposición del personal de la institución. El análisis de los datos se realizará de manera centralizada en el Departamento de Estadística Médica, de la Universidad Médica de Viena, en Austria. Este estudio permite establecer los puntos de referencia a nivel nacional con instituciones comparables de todo el mundo. Para conocer más sobre el nutritionDay, consulte la página web: https://www.nutritionday.org/.

El nutritionDay en el mundo tiene como punto de partida la Resolución sobre la atención alimentaria y nutricional en los hospitales del Consejo de Europa de $2003^{(2)}$. En Colombia se alinea con la Declaración
\end{abstract}

Coordinadora Nacional del nutritionDay. Asociación Colombiana de Nutrición Clínica.

angelica_perezc@hotmail.com de Cartagena sobre el derecho al cuidado nutricional y la lucha contra la malnutrición ${ }^{(3)}$. El estudio cuenta con el respaldo de la Sociedad Europea de Nutrición Clínica y Metabolismo (ESPEN), la Sociedad Austríaca de Nutrición Clínica (AKE), la Universidad Médica de Viena (MUW), así como la Sociedad Alemana de Medicina Nutricional (DGEM). El centro de coordinación está en Viena, Austria. En Colombia, bajo el liderazgo de Angélica Pérez, la Asociación Colombiana de Nutrición Clínica (ACNC) promueve, coordina, acompaña y vigila el correcto desarrollo de la jornada. Además, se encarga de la transferencia de la información a la sede central del nutritionDay en Viena bajo un código y una clave asignada por la organización nutritionDay.

Colombia ha participado en el nutritionDay de manera ininterrumpida desde 2009. Recientemente, hemos publicado el análisis de 7 años de participación en el estudio (2009-2015). El análisis de los datos de 7994 pacientes hospitalizados en instituciones colombianas, 7243 en Latinoamérica y 155524 en el mundo, permitió mostrar que la prevalencia del riesgo de desnutrición fue de $38 \%, 41 \%$ y $32 \%$, respectivamente. De los pacientes en riesgo de desnutrición en Colombia, únicamente el $23 \%$ recibe algún tratamiento nutricional ${ }^{(4)}$. El estudio mostró también que los pacientes con riesgo de desnutrición tienen el doble de probabilidad de morir (Hazard ratio $[\mathrm{HR}] 1,94$; intervalo de confianza [IC] del $95 \%$, $1,53,2,46 ; p<0,001)$ y menor probabilidad de egreso al domicilio (HR 0,82; IC del $95 \%, 0,76,0,88, p<0,001$ ).

A partir de estos resultados, la ACNC pretende llegar a las entidades gubernamentales y desarrollar políticas y programas que busquen promover una cultura institucio- 
nal, la cual tenga en cuenta el cuidado nutricional. De esta forma, se busca que se respete el derecho fundamental al cuidado nutricional y, por ende, que todos lo pacientes reciban una terapia nutricional óptima y oportuna.

Este año, una vez más, Colombia le cumple al nutritionDay. La ACNC coordinó y acompañó el proceso de inscripción, capacitación y organización de 28 instituciones colombianas y 18 instituciones de Perú, Ecuador, Guatemala y Panamá. Estas instituciones lograron superar las dificultades propias de la pandemia y, motivadas

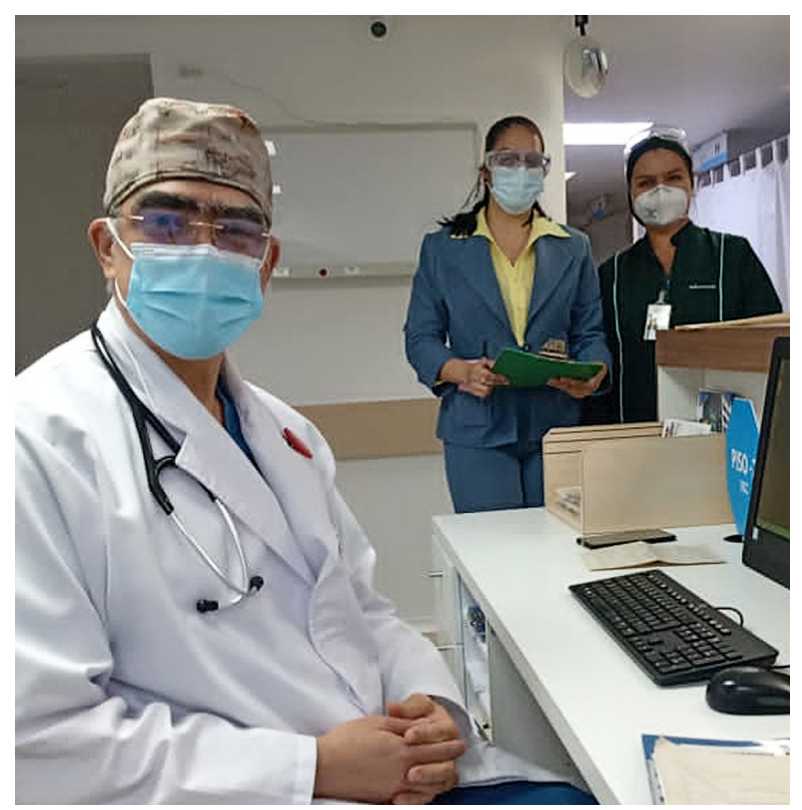

Figura 1. Grupo de Soporte Nutricional, Clínica Especializada EMMSA, Medellín, Colombia.

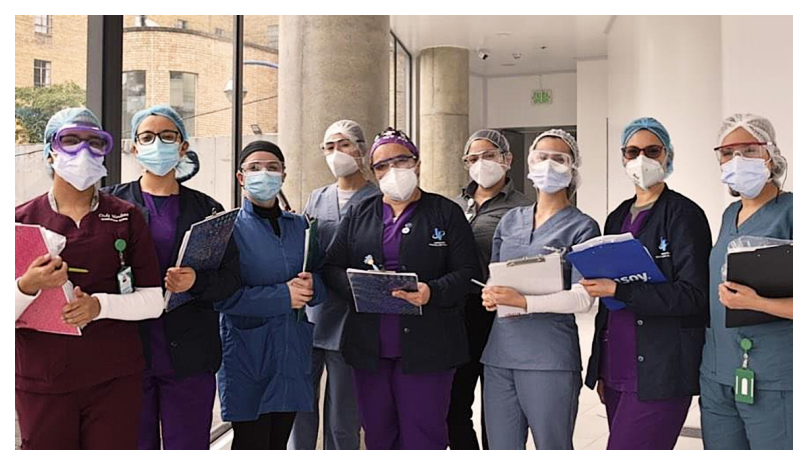

Figura 2. Grupo de Nutrición Clínica, Fundación Hospital San Carlos, Bogotá, Colombia. por el deseo de trabajar por la nutrición en los hospitales, participaron en el nutritionDay el 12 de noviembre. La información de los pacientes con COVID-19 fue recolectada, lo que nos permitirá tener una idea del cuidado nutricional de estos pacientes.

Queremos dar las gracias a cada uno de los encuestadores e instituciones que ven como una estrategia de mejoramiento continuo y una oportunidad de mejora la realización del nutritionDay en sus instituciones (Figuras 1-4).

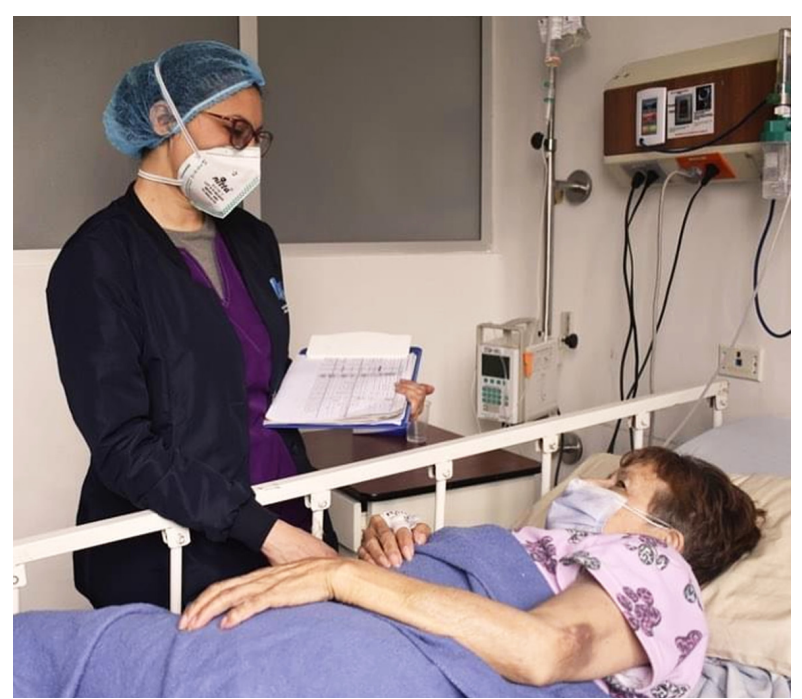

Figura 3. Nutricionista clínica, Fundación Hospital San Carlos. Bogotá, Colombia.

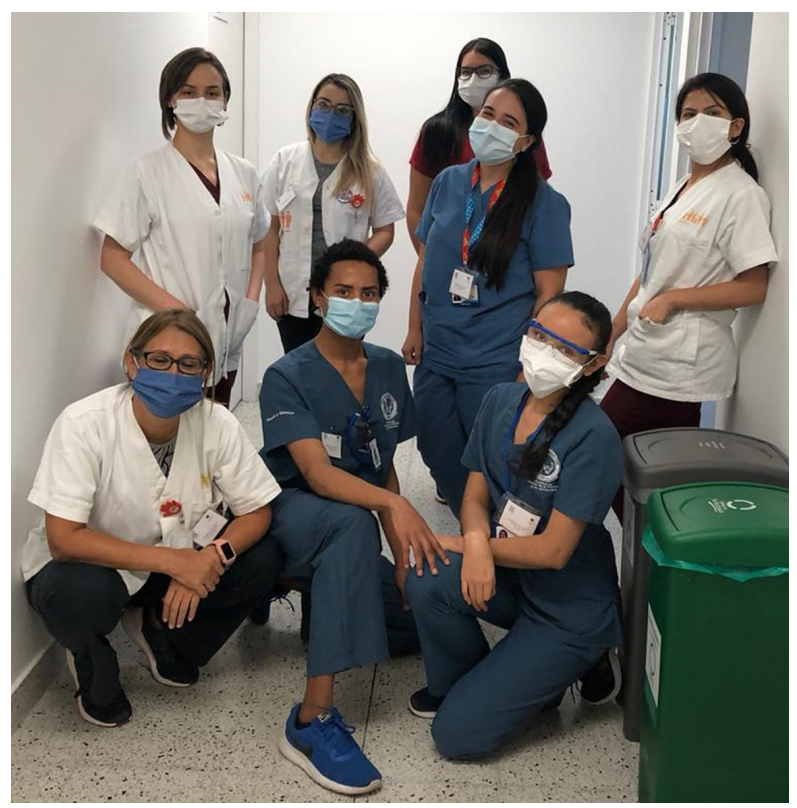

Figura 4. Grupo de Nutrición Clínica, Hospital General de Medellín, Colombia. 


\section{Referencias bibliográficas}

1. Schindler K, Pichard C, Sulz I, Volkert D, Streicher M, Singer P. nutritionDay: 10 years of growth. Clin Nutr. 2017;36(5):1207-1214. doi: 10.1016/j.clnu.2016.11.004.

2. Council of Europe Committee of Ministers. Resolution resap(2003)3 on food and nutritional care in hospitals [Internet]. Strasbourg (France); 2003. (Consultado el 1 de diciembre 2020) Disponible en: https://www.nutritionday. $\mathrm{org} / \mathrm{cms} / \mathrm{upload} / \mathrm{pdf} / 11$.resolution/Resolution_of_the Council_of_Europe.pdf.
3. Cárdenas D, Bermúdez Ch, Echeverri S, Pérez A, Puentes M, López L, et al. Declaración Internacional sobre el Derecho al Cuidado nutricional y la Lucha contra la Malnutrición. Rev Nutr Clin Metab. 2019;2(Supl1), 14-23. doi: 10.35454/rncm. v2supl1.015.

4. Cárdenas D, Bermúdez C, Pérez A, Díaz G, Cortes LY, Contreras $\mathrm{CP}$, et al. Nutritional risk is associated with an increase of in-hospital mortality and a reduction of being discharged home: Results of the 2009-2015 nutritionDay survey. Clin Nutr ESPEN. 2020;38:138-145. doi: 10.1016/j. clnesp.2020.05.014. 